Case report

\title{
Impingement syndrome of the shoulder following double row suture anchor technique for arthroscopic rotator cuff repair: a case report Rohit Rambani $^{1 *}$ and Roger G Hackney ${ }^{2}$
}

Addresses: ${ }^{1}$ Trauma and Orthopaedics, Leeds Teaching Hospital NHS Trust, Leeds, UK and ${ }^{2}$ Chapel Allerton Orthopaedic Hospital, Leeds, UK Email: RH* - rohitrambani@gmail.com

* Corresponding author

Received: 27 September 2008 Accepted: 12 March 2009 Published: 12 June 2009

Journal of Medical Case Reports 2009, 3:8109 doi: 10.4076/1752-1947-3-8109

This article is available from: http://jmedicalcasereports.com/jmedicalcasereports/article/view/8109

(C) 2009 Rambani and Hackney; licensee Cases Network Ltd.

This is an Open Access article distributed under the terms of the Creative Commons Attribution License (http://creativecommons.org/licenses/by/3.0), which permits unrestricted use, distribution, and reproduction in any medium, provided the original work is properly cited.

\begin{abstract}
Introduction: Arthroscopic repair of the rotator cuff is a demanding surgery. Accurate placement of anchors is key to success.

Case presentation: A 38-year-old woman received arthroscopic repair of her rotator cuff using a double row suture anchor technique. Postoperatively, she developed impingement syndrome which resulted from vertical displacement of a suture anchor once the shoulder was mobilised. The anchor was removed eight weeks following initial surgery and the patient had an uneventful recovery.

Conclusion: Impingement syndrome following arthroscopic repair of the rotator cuffs using double row suture anchor has not been widely reported. This is the first such case where anchoring has resulted in impingement syndrome.
\end{abstract}

\section{Introduction}

Arthroscopic repair of a rotator cuff tear is a demanding technique. Arthroscopic repairs of rotator cuff tears have become more popular than open or mini-open repairs $[1,2]$. The use of double row suture anchor technique has become the standard technique among many arthroscopic shoulder surgeons [3]. Recent reports have suggested high percentages of good to excellent results even for large or massive tears with 1-3 years follow up. But the literature is still not clear about the long-term results comparing open repairs with arthroscopic repairs $[2,4]$. The influence of the repair technique on the failure rates and functional outcomes after open or arthroscopic rotator cuff repair remains controversial [5].
Acute impingement syndrome following rotator cuff repair has been reported to occur due to heterotopic ossification [6]. There have been no reports of acute impingement syndrome resulting from arthroscopic repair of rotator cuff from a suture anchor.

\section{Case presentation}

A 38-year-old woman presented with a traumatic tear of her right supraspinatus measuring $1.2 \mathrm{~cm}$ in length and $8 \mathrm{~mm}$ in transverse diameter. It was repaired arthroscopically using a double row suture anchor technique. A metallic FASTIN suture anchor with orthocord (Depuy Inc. USA) was used initially followed by the Quick-T anchor fixation system (Smith \& Nephew Inc Switzerland). The 


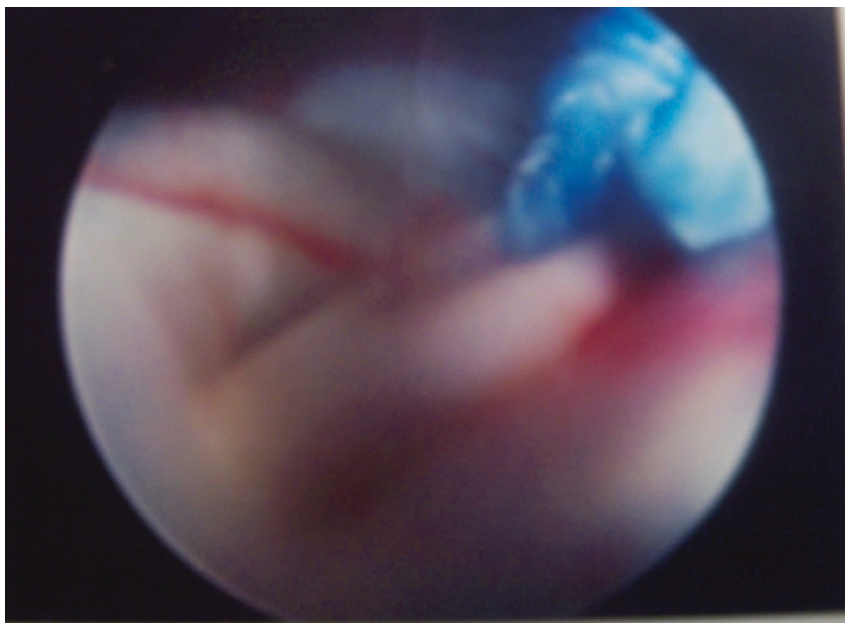

Figure I. Intra-operative photograph showing the button placed correctly.

initial post-operative procedure was uneventful. The patient's shoulder was mobilized at six weeks. The patient started complaining of pain on abduction. This was initially treated with analgesics but when the patient did not settle the shoulder was examined using ultrasound. This showed inflammatory changes in the subacromial space and was inconclusive. The intra-operative photographs of the repair did not show any abnormality or evidence of impingement syndrome (Figure 1). The patient was taken back to theatre for diagnostic arthroscopy of the shoulder which showed the button of the Quick-T anchor lying vertically in the subacromial space rubbing the undersurface of the acromion (Figure 2). This was removed using a shaver without compromising the

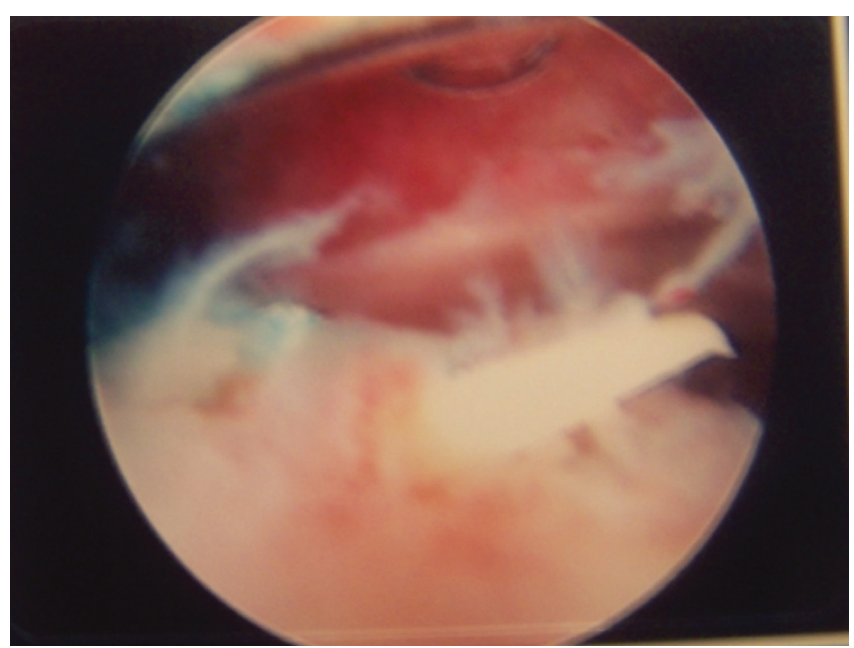

Figure 2. Intra-operative photograph showing the button placed vertically ribbing the undersurface of acromion. repair. Subacromial decompression was not required as there was no other evidence of impingement. Postoperatively the pain completely resolved. At follow-up three months after the second arthroscopy, the patient had fullrange shoulder movement and pain was no longer present.

\section{Discussion}

The main causes of postoperative shoulder pain include frozen shoulder [7], failure of repair [8], reflex sympathetic dystrophy [9] and subcoracoid impingement syndrome [10]. Many papers have discussed the readmission and reoperation rate after rotator cuff repairs [8] but the incidence of subacromial impingement is not documented in patients with no impingement preoperatively or intraoperatively.

The shoulder's subacromial space is of significant clinical interest due to its association with rotator cuff disease. Recent trials have suggested that subacromial decompression did not seem to significantly affect the outcome of arthroscopic rotator cuff repair on shorter follow-ups [4]. Post-operative diagnosis is usually inconclusive using ultrasonography when postoperative impingement is suspected because of inflammatory changes in the subacromial space because of surgery.

The introduction of rotator cuff MITEK anchors brought forth a fairly exclusive procedure for refixation of rotator cuff ruptures [11]. The reinsertion of the rotator cuff to their bony footprints has been suggested to have stronger and quicker healing $[11,12]$. Bay et al. reported an in vivo increase in subacromial space after rotator cuff repair [13].

\section{Conclusion}

Impingement syndrome due to Quick-T suture anchors has not been reported in the literature. We report an interesting and unusual case of impingement syndrome following double row suture anchor technique.

\section{Competing interests}

The author(s) declare that they have no competing interests.

\section{Consent}

Written informed consent was obtained from the patient for publication of this case report and accompanying images. A copy of the written consent is available for review by the Editor-in-Chief of this journal.

\section{References}

I. lannotti JP: Full-Thickness Rotator Cuff Tears: Factors Affecting Surgical Outcome. J Am Acad Orthop Surg 1994, 2:87-95.

2. Sugaya $\mathrm{H}$ et al.: Functional and structural outcome after arthroscopic full-thickness rotator cuff repair: single-row versus dual-row fixation. Arthroscopy 2005, 2 I: I307-1316. 
3. Lafosse $L$ et al.: The outcome and structural integrity of arthroscopic rotator cuff repair with use of the double-row suture anchor technique. J Bone Joint Surg Am 2007, 89: I533-I54I.

4. Boileau $P$ et al.: Arthroscopic repair of full-thickness tears of the supraspinatus: does the tendon really heal? J Bone Joint Surg Am 2005, 87: 1229-1240.

5. Bishop J et al.: Cuff integrity after arthroscopic versus open rotator cuff repair: a prospective study. J Shoulder Elbow Surg 2006, I 5:290-299.

6. Kircher J, Martinek V, Mittelmeier W: Heterotopic ossification after minimally invasive rotator cuff repair. Arthroscopy 2007, 23: 1359 el-3.

7. Boszotta $\mathrm{H}$, Prunner K: Arthroscopically assisted rotator cuff repair. Arthroscopy 2004, 20:620-626.

8. Green LB et al.: Sources of variation in readmission rates, length of stay, and operative time associated with rotator cuff surgery. J Bone Joint Surg Am 2003, 85A: I784-1789.

9. Brislin KJ, Field LD, Savoie FH 3rd: Complications after arthroscopic rotator cuff repair. Arthroscopy 2007, 23:124-128.

10. Dines $D M$ et al.: The coracoid impingement syndrome. J Bone Joint Surg Br 1990, 72:3।4-3I6.

II. Barber FA, Cawley P, Prudich JF: Suture anchor failure strengthan in vivo study. Arthroscopy 1993, 9:647-652.

12. Park $M C$ et al.: Tendon-to-bone pressure distributions at a repaired rotator cuff footprint using transosseous suture and suture anchor fixation techniques. Am J Sports Med 2005, 33:1154-1159.

13. Bey MJ et al.: In vivo measurement of subacromial space width during shoulder elevation: technique and preliminary results in patients following unilateral rotator cuff repair. Clin Biomech (Bristol, Avon) 2007, 22:767-773.

\section{Do you have a case to share?}

Submit your case report today

- Rapid peer review

- Fast publication

- PubMed indexing

- Inclusion in Cases Database

Any patient, any case, can teach us something

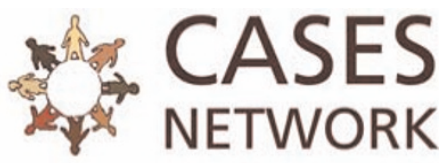

www.casesnetwork.com 